\title{
Nephroprotective effect of magnesium isoglycyrrhizinate against arsenic trioxide-induced acute kidney damage in mice
}

\author{
ZIHENG WEI $^{1^{*}}$, XIAOQI SUN ${ }^{1 *}$, QIANQIAN HE ${ }^{1}$, YANG ZHAO $^{2}$, YONGCHAO WU $^{3}$, \\ $\mathrm{XUE} \mathrm{HAN}^{1,4}, \mathrm{ZHONGLIN} \mathrm{WU}^{3}, \mathrm{XI} \mathrm{CHU}^{5}$ and SHENGJIANG GUAN ${ }^{6,7}$ \\ ${ }^{1}$ School of Pharmacy, Hebei University of Chinese Medicine, Shijiazhuang, Hebei 050200; \\ Departments of ${ }^{2}$ Academic Research and ${ }^{3}$ Radiological Intervention, The Fourth Hospital of Hebei Medical University, \\ Shijiazhuang, Hebei 050011; ${ }^{4}$ Hebei Key Laboratory of Integrative Medicine on Liver-Kidney Patterns, Shijiazhuang, \\ Hebei 050200; ${ }^{5}$ Department of Pharmacy, The Fourth Hospital of Hebei Medical University, Shijiazhuang, Hebei 050011; \\ ${ }^{6}$ School of Basic Medicine, Hebei University of Chinese Medicine; ${ }^{7}$ Department of Pharmacy, \\ Affiliated Hospital, Hebei University of Chinese Medicine, Shijiazhuang, Hebei 050200, P.R. China
}

Received November 16, 2021; Accepted January 17, 2022

DOI: $10.3892 /$ etm.2022.11202

\begin{abstract}
Magnesium isoglycyrrhizinate (MgIG) has antiinflammatory, antioxidative, antiviral and anti-hepatotoxic effects. However, protective effects of $\mathrm{MgIG}$ against renal damage caused by arsenic trioxide (ATO) have not been reported. The present study aimed to clarify the protective function of MgIG on kidney damaged induced by ATO. Other than the control group and the group treated with $\mathrm{MgIG}$ alone, mice were injected intraperitoneally with ATO $(5 \mathrm{mg} / \mathrm{kg} / \mathrm{day})$ for 7 days to establish a mouse model of kidney damage. On the 8th day, blood and kidney tissue were collected and the inflammatory factors and antioxidants levels in the kidney tissue and serum were measured. The expression of protein levels of caspase-3, Bcl-2, Bax, Toll-like receptor-4 (TLR4) and nuclear factor- $\kappa \mathrm{B}(\mathrm{NF}-\kappa \mathrm{B})$ were determined via western blot analysis. In the renal tissue of mice, ATO exposure dramatically elevated markers of oxidative stress, apoptosis and inflammation. However, MgIG could also restore the activities of urea nitrogen and creatinine to normal levels, decrease the malondialdehyde level and reactive oxygen species formation and increase superoxide dismutase, catalase and glutathione
\end{abstract}

Correspondence to: $\mathrm{Dr} \mathrm{Xi}$ Chu, Department of Pharmacy, The Fourth Hospital of Hebei Medical University, 12 Jiankang Road, Shijiazhuang, Hebei 050011, P.R. China

E-mail: chux2014@126.com

Professor Shengjiang Guan, School of Basic Medicine, Hebei University of Chinese Medicine, 326 Xinshi South Road, Shijiazhuang, Hebei 050200, P.R. China

E-mail: guanshengjiang123@126.com

${ }^{*}$ Contributed equally

Key words: magnesium isoglycyrrhizinate, arsenic trioxide, oxidative stress, TLR4/NF- $\mathrm{B}$ signaling pathway activities. MgIG also ameliorated the morphological abnormalities generated by ATO, reduced inflammation and apoptosis and inhibited the TLR4/NF- $\kappa$ B signaling pathway. In conclusion, $\mathrm{MgIG}$ may mitigate ATO-induced kidney damage by decreasing apoptosis, oxidative stress and inflammation and its mechanism may be connected to the inhibition of TLR4/NF- $\mathrm{B}$ signaling.

\section{Introduction}

Throughout history, arsenic has been recognized as both an environmental hazard and a therapeutic chemical (1). Arsenic trioxide (ATO) is widely considered an effective anti-cancer treatment medicine (2) and it is the leading treatment medicine for acute promyelocytic leukemia. Human populations exposed to high level of arsenic are at risk of developing skin, bladder, liver and lung cancers (3). Despite the well-known toxicity of ATO, it has been used to treat various diseases for centuries (4).

The kidney is an important organ that not only can maintain blood pressure and eliminate waste, but also can maintain and regulate body fluid, such as acid-base balance (5). Kidney damage or nephrotoxicity leads to the impairment of detoxification and excretion functions, which can be confirmed by renal markers such as blood urea nitrogen (BUN), creatinine (CRE) and CRE clearance (6).

Research has shown that ATO can cause kidney damage. Indeed, oxidation, inflammation and apoptosis have all been linked to ATO-induced nephrotoxicity $(7,8)$. The primary mechanism by which ATO causes nephrotoxicity is oxidative stress (9). Additionally, oxidative stress can increase reactive oxygen species (ROS) production and malondialdehyde (MDA) level while decreasing superoxide dismutase (SOD), catalase (CAT) and glutathione (GSH) activities, leading to apoptosis $(10,11)$. Thus, antioxidative agents may mitigate or prevent ATO-induced nephrotoxicity.

Toll-like receptor-4 (TLR4) acts as a lipopolysaccharide sensor to induce inflammation by activating factors that induce 
inflammation (12). TLR4 may activate the intercellular nuclear factor- $\kappa \mathrm{B}(\mathrm{NF}-\kappa \mathrm{B})$, given the relationship between innate and acquired immunity (13). The NF- $\kappa \mathrm{B}$ pathway is further activated, which leads to the entry of the p-p65 protein into the nucleus and increases the expressions of the pro-inflammatory factors $(14,15)$.

ATO is capable of stimulating the formation of ROS (16). Overproduction of ROS is thought to activate the NF- $\kappa B$ pathway and result in the upregulation of pro-inflammatory mediators $(17,18)$. On the whole, increased oxidative stress and consequent inflammation may result in a greater number of cells undergoing apoptosis (19). NF- $\kappa \mathrm{B}$ is implicated in regulating numerous inflammatory genes and ATO-induced various stimuli can stimulate NF- $\mathrm{NB}$ (20). In conclusion, oxidative stress is recognized to serve a significant role in ATO-induced toxicity (21).

Magnesium isoglycyrrhizinate (MgIG; Fig. 1) is derived from 18-glycyrrhizic acid, which is a major component of natural licorice (Glycyrrhiza glabra L.) and has been shown to protect the liver and possesses anti-inflammatory, anti-oxidative and anti-apoptotic properties $(22,23)$. In pharmacological experiments, MgIG has been demonstrated to suppress the inflammatory process, decrease pathological damage to hepatocytes and enhance the overall function of hepatocytes (24). Furthermore, MgIG protects the heart by inhibiting the TLR4/NF- $\kappa \mathrm{B}$ signaling pathway, which is responsible for activating inflammatory factors (25). Licorice and its active components have been found to be protective against kidney damage (26). Nevertheless, no studies to date have examined the possible effects and mechanisms of MgIG on the nephrotoxicity caused by ATO.

The present study constructed a mouse model of ATO-induced kidney injury and examined the effects of MgIG on renal morphological and renal function to investigate the effects and potential mechanisms of MgIG in ATO-treated mice. In addition, the regulation of $\mathrm{MgIG}$ in the TLR4/NF- $\mathrm{BB}$ signaling pathways was investigated.

\section{Materials and methods}

Chemicals and drugs. MgIG for injection was purchased from the Chia Tai Tianqing Pharmaceutical Group Co., Ltd. ATO parenteral solution was purchased from Beijing SL Pharmaceutical Co., Ltd. All additional analytical-grade reagents were acquired from MilliporeSigma.

Animals. A total of 50 male Kunming mice (weight: $22.0 \pm 2.0 \mathrm{~g}$, age: 6-7 weeks) were acquired from the Hebei Medical University Center for Laboratory Animals. The mice were fed at a normative temperature $\left(22 \pm 2^{\circ} \mathrm{C}\right)$ and humidity $(50 \pm 10 \%)$. The mice were fed a standard pellet diet and water ad libitum. Animal studies at Hebei Medical University of Chinese Medicine (Shijiazhuang, China) were conducted in compliance with the Animal Care and Ethical Committee (approval no. DWLL2020005) and the United Kingdom Animal (Scientific Procedures) Act 1986. The Reporting of In Vivo Experiments guidelines were used to guide the current study (27).

Experimental design. Male mice were randomly divided into 5 groups $(\mathrm{n}=10)$ : The control group (CONT; saline i.p. $10 \mathrm{mg}$ / $\mathrm{kg} /$ day), the ATO group (ATO; i.p. $5 \mathrm{mg} / \mathrm{kg} /$ day) $(28,29)$, the MgIG only group (MgIG; i.p. $50 \mathrm{mg} / \mathrm{kg} /$ day), the low-dose MgIG group (L-MgIG; i.p. $25 \mathrm{mg} / \mathrm{kg} / \mathrm{day})(30,31)$ and the high-dose MgIG group (H-MgIG; i.p. $50 \mathrm{mg} / \mathrm{kg} /$ day). With the exception of the CONT and MgIG groups, all mice were injected i.p. with ATO (5 mg/kg/day) for a total of 7 days. All 50 mice were still alive during the experiment and the body weight and status of the mice were checked daily. The criteria of humane endpoints for euthanasia were body weight loss $(>10 \%)$ and anorexia in contrast with the controls. Following the last administration, the urine of the mice in each group was collected, after which the mice were anesthetized with sodium pentobarbital $(50 \mathrm{mg} / \mathrm{kg})$ and blood was drawn from the eyeballs. Then, the mice were sacrificed by intraperitoneal injection of an overdose of sodium pentobarbital $(200 \mathrm{mg} / \mathrm{kg})(28,32)$. Animal death was confirmed by the loss of signs of life, such as toe pinch response, heartbeat and breathing. After mice were sacrificed, kidney tissues were rapidly collected for subsequent experiments.

Urine and serum chemistry analysis. Blood (3,500 x g; $10 \mathrm{~min}$; $\left.4^{\circ} \mathrm{C}\right)$ and urine $\left(1,500 \times \mathrm{g} ; 5 \mathrm{~min} ; 4^{\circ} \mathrm{C}\right)$ samples were obtained by centrifugation. The supernatant was then collected into Doff tubes. The serum and urine were refrigerated at $-20^{\circ} \mathrm{C}$ until use. The expression activities of BUN (cat. no. C013-2-1; Jiancheng Institute of Bioengineering) and CRE (cat. no. C011-2-1; Jiancheng Institute of Bioengineering) were determined by using colorimetric assay. Creatinine clearance was calculated according to the equation: Creatinine clearance $(\mu \mathrm{l} / \mathrm{min} / \mathrm{g}$ body weight $)=[$ urinary $\operatorname{CRE}(\mu \mathrm{mol} / \mathrm{l}) \times$ urine volume $(\mu 1 / \mathrm{min}) /$ serum CRE $(\mu \mathrm{mol} / \mathrm{l}) \mathrm{x}$ body weight $(\mathrm{g})](33,34)$.

Histopathological analysis. The kidney samples of mice were bisected, trimmed and fixed in $10 \%$ formalin at room temperature for $48 \mathrm{~h}$. The samples were dehydrated in graded ethanol concentrations and then embedded in paraffin. Paraffin sections (4- $\mu \mathrm{m})$ were stained with hematoxylin for $5 \mathrm{~min}$ and eosin for 3 min at room temperature. Light microscopy (Leica DM4000B; Leica Microsystems $\mathrm{GmbH}$ ) was used to examine histological alterations. Score 1, 2, 3, 4 and 5 represent the kidney injury area $<10,10-25,25-50,50-75$ and $>75 \%$, respectively. The renal injury was assessed by scoring tubular cell swelling, cellular vacuolization and others under the light microscopy in $\geq 10$ different horizons and the average score calculated.

Measurement of oxidative stress and antioxidant enzymes. The collected serum and kidney tissues were used to detect the activities of GSH, SOD, CAT, MDA. The expression activities of GSH (cat. no. A006-2-1; Jiancheng Institute of Bioengineering), SOD (cat. no. A001-3-2; Jiancheng Institute of Bioengineering), CAT (cat. no. A007-1-1; Jiancheng Institute of Bioengineering) and MDA (cat. no. A003-1-1; Jiancheng Institute of Bioengineering) were determined by using colorimetric assay.

Measurement of ROS levels in the kidney. Ethoxylation is commonly used as a method to monitor the production of reactive ROS in cells (35). Dihydroethidium (DHE; cat. no. G10451 Wuhan Servicebio Technology Co., Ltd.) fluorescence was used to evaluate ROS production in the kidney. The kidney specimens were flash-frozen in liquid nitrogen. Then, the 


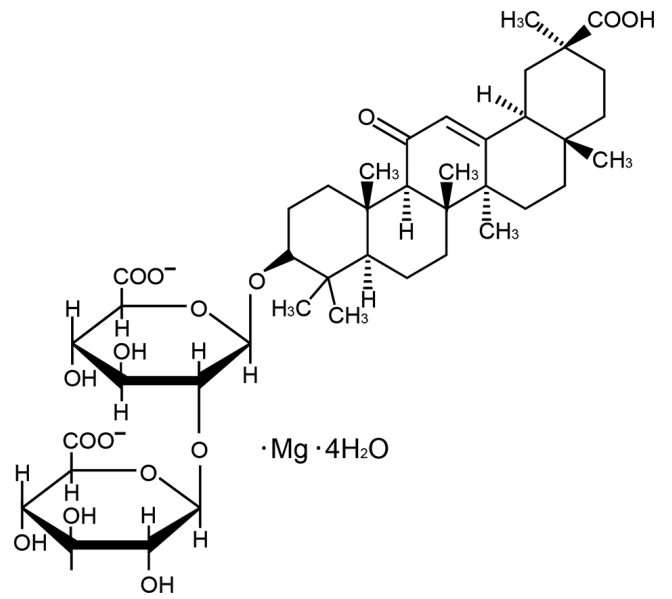

Figure 1. Chemical construction of magnesium isoglycyrrhizinate.

freezing microtome (Cryotome E; Thermo Fisher Scientific, Inc.) was used to cut slices. Frozen slices $(5-\mu \mathrm{m})$ were incubated for $5 \mathrm{~min}$ with a spontaneous fluorescence-quenching reagent. The slides were then rinsed for $10 \mathrm{~min}$ with flowing water and the DHE was then dropped into the indicated region and incubated for $30 \mathrm{~min}$ at $37^{\circ} \mathrm{C}$ in the dark. After washing with PBS ( $\mathrm{pH} 7.4$ ) in a Rocker device, the slides were incubated in a dark location with a DAPI solution for $10 \mathrm{~min}$ at room temperature. A total of 10 microscopic fields in each section were examined in a blinded method. A fluorescence microscope (Nikon Eclipse C1; Nikon Corporation) was used to observe and capture images at x200 magnification.

Inflammatory cytokine analysis. The kidney samples were promptly snap-frozen at $-196^{\circ} \mathrm{C}$ in liquid nitrogen for standby use. Interleukin-6 (IL-6; cat. no. 88-7064-88; MultiSciences Biotech Co., Ltd.), interleukin-1 $\beta$ (IL-1 $\beta$; cat. no. 88-7013-88; MultiSciences Biotech Co., Ltd.) and tumor necrosis factor alpha (TNF- $\alpha$; cat. no. 88-7324-88; Thermo Fisher Scientific, Inc.) levels in the kidney were measured by relevant ELISA kits.

Bax, Bcl-2, Caspase-3, TLR4, NF- $\kappa B$ and $p-N F-\kappa B$ by western blotting analysis. RIPA lysis buffer (Wuhan Servicebio Technology Co., Ltd.) was used to decompose the kidney samples. Centrifugation $\left(12,000 \mathrm{x} \mathrm{g} ; 10 \mathrm{~min} ; 4^{\circ} \mathrm{C}\right)$ was used to collect the total protein in tissue that concentration was determined with the BCA kit (catalog no. G2026; Wuhan Servicebio Technology Co., Ltd.). Sodium dodecyl sulfate polyacrylamide gel electrophoresis (10\%) was used to separate equal amounts of protein $(15 \mu \mathrm{g})$. The proteins were transferred to polyvinylidene difluoride membranes that were soaked for overnight at $37^{\circ} \mathrm{C}$ in a blocking solution containing $5 \%$ skimmed milk. Then, the membranes were incubated with anti-Bcl-2 associated $\mathrm{X}$ protein (Bax; cat. no. GB11690, 1:1,000; Wuhan Servicebio Technology Co., Ltd.), anti-B-cell lymphoma 2 (Bcl-2; cat. no. PAA778Mu01, 1:1,000; Cloud-Clone Corp.), anti-caspase-3 (cat. no. 66470-2-lg, 1:1,000; ProteinTech Group, Inc.), anti-NF- $\mathrm{B}$ (cat. no. GM1003, 1:2,000; Wuhan Servicebio Technology Co., Ltd.), anti-p-NF-кB (cat. no. GB11142-1, 1:2,000; Wuhan Servicebio Technology Co., Ltd.), anti-TLR4 (cat. no. GB11519, 1:1,000; Wuhan Servicebio Technology Co., Ltd.) and $\beta$-actin (cat. no. GB12001, 1:1,000; Wuhan Servicebio Technology Co., Ltd.) antibodies at $4^{\circ} \mathrm{C}$ for
$12 \mathrm{~h}$. Afterwards, the membranes were rinsed three times with PBS. Membranes were incubated for $2 \mathrm{~h}$ at $37^{\circ} \mathrm{C}$ in darkness with secondary antibodies of goat anti-mouse IgG-HRP (cat. no. GB23301; 1:3,000; Wuhan Servicebio Technology Co., Ltd.) or goat anti-rabbit IgG-HRP (cat. no. GB23303; 1:3,000; Wuhan Servicebio Technology Co., Ltd.) to detect the protein bands. After subjecting the membranes to a chemiluminescent substrate (ECL; catalog no. G2014; Wuhan Servicebio Technology Co., Ltd.) and to autoradiographic film exposure, the optical density value of the target band was analyzed using Alpha software (Alpha Innotech).

Statistical analysis. Origin Pro 9.1 (OriginLab Corp.) software was used to perform statistical analyses. Data are demonstrated as mean \pm SEM and one-way analysis of variance (ANOVA) and Tukey's post hoc test were used to assess statistically significant differences between groups. Non-parametric data were analyzed by the Kruskal-Wallis test and the Dunn-Sidak post hoc test (36). $\mathrm{P}<0.05$ was considered to indicate a statistically significant difference.

\section{Results}

Effects of MgIG on alterations in biochemical indices. In Fig. 2, the levels of serum BUN, serum CRE, urinary CRE and $\mathrm{CRE}$ clearance were measured to examine the effect of MgIG on ATO-induced nephrotoxicity. Serum BUN and CRE levels were notably augmented and urinary $\mathrm{CRE}$ and CRE clearance levels were reduced in the ATO group $(\mathrm{P}<0.01)$ compared with the CONT group. However, the serum BUN and CRE levels of the H-MgIG and L-MgIG groups were markedly decreased but urinary CRE and CRE clearance levels were augmented in contrast with the ATO group $(\mathrm{P}<0.05$ or $\mathrm{P}<0.01)$. There was no significant difference in levels of serum BUN, serum CRE, urinary CRE and CRE clearance between the CONT group and the MgIG group ( $\mathrm{P}>0.05)$. MgIG may have an effect of renal protection.

Effects of MgIG on the histopathological changes. As shown in Fig. 3, light microscopy was used to visualize hematoxylin and eosin-stained kidney slices. No morphological changes were found in the kidney tissues of the CONT and MgIG groups. By contrast, the ATO group showed tubular cell swelling, interstitial edema, inflammatory cell infiltration in the renal interstitial tissue, glomeruli dilation and hyperemia, partial epithelial cell necrosis $(\mathrm{P}<0.01)$. The mice who received $\mathrm{H}-\mathrm{MgIG}$ and L-MgIG treatment had reduced areas of necrosis and inflammatory infiltration $(\mathrm{P}<0.05$ or $\mathrm{P}<0.01)$. In other words, treatment of MgIG may ameliorated ATO-induced kidney damage in a dose-dependent manner.

Effects of MgIG on the expression levels of antioxidant enzymes. As shown in Figs. 4 and 5, the ATO group showed markedly decreased the activities of SOD, CAT and GSH in serum and kidney tissues $(\mathrm{P}<0.01)$ and the content of MDA was increased, in contrast with the CONT group. Compared with the ATO group, the activities of SOD, CAT and GSH in the groups of MgIG therapy were enhanced $(\mathrm{P}<0.05$ or $\mathrm{P}<0.01)$ and the level of MDA was decreased $(\mathrm{P}<0.01)$. In contrast to the control group, mice that only received $50 \mathrm{mg}$ / 
A

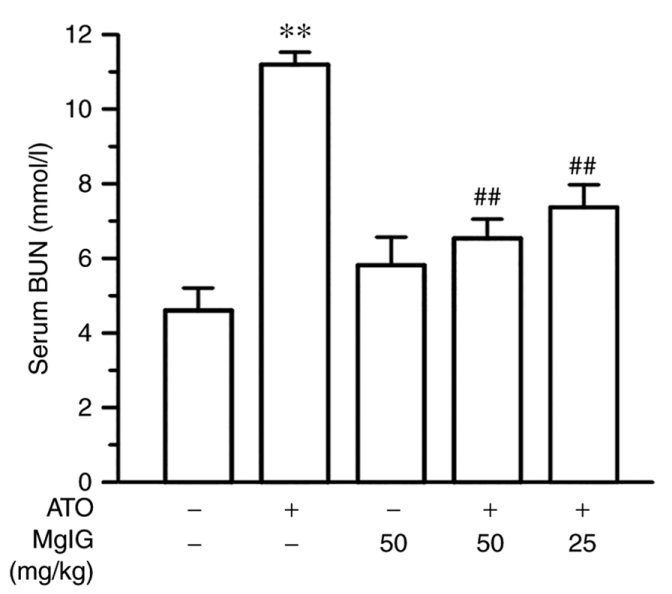

C

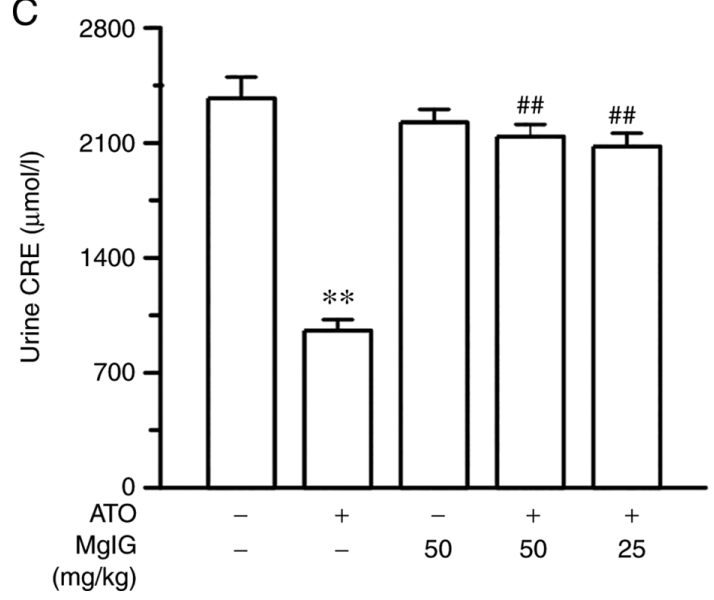

B

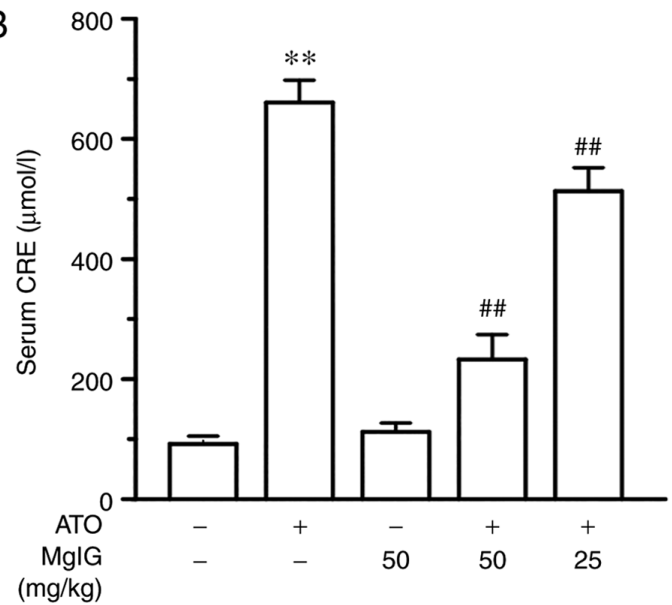

D

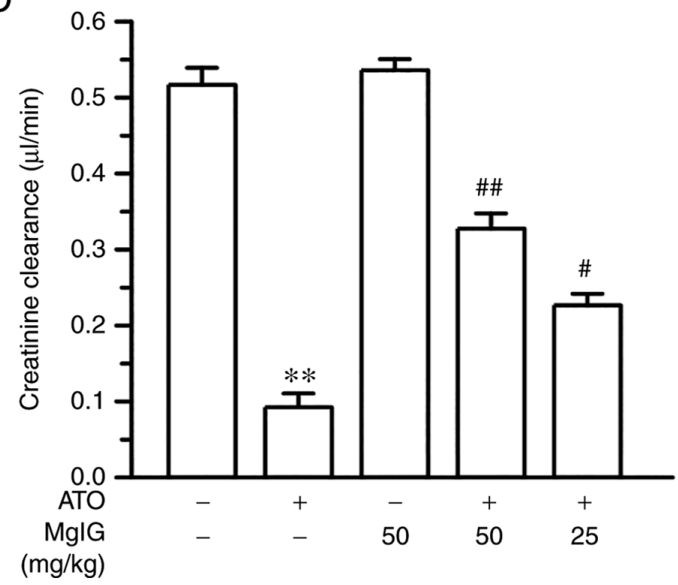

Figure 2. Effects of MgIG on levels of serum (A) BUN and (B) CRE and urine (C) CRE and (D) creatinine clearance. Values are demonstrated as mean \pm SEM, $\mathrm{n}=10 .{ }^{* *} \mathrm{P}<0.01$ vs. CONT group; ${ }^{\# \#} \mathrm{P}<0.01,{ }^{*} \mathrm{P}<0.05$ vs. ATO group. MgIG, magnesium isoglycyrrhizinate; BUN, blood urea nitrogen; CRE, creatinine; CONT, control group; ATO, arsenic trioxide group.

A

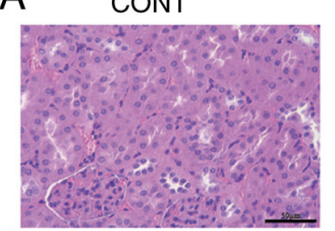

ATO

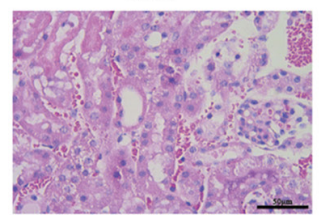

ATO

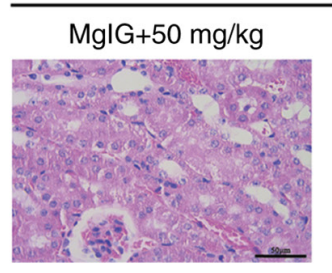

$$
\mathrm{MglG}+25 \mathrm{mg} / \mathrm{kg}
$$

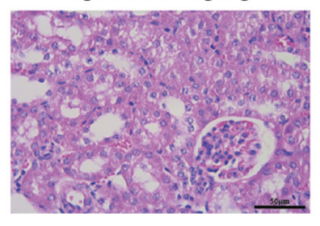

$\mathrm{MglG}$

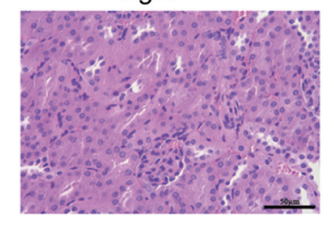

B

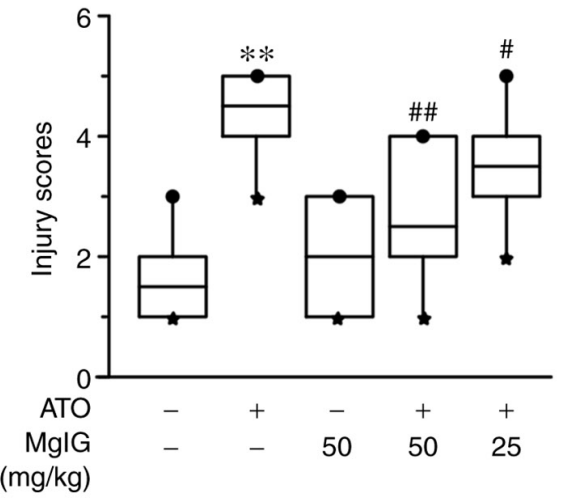

Figure 3. Effects of MgIG on histopathological variations in mice, as observed by hematoxylin and eosin staining [(A) magnification, $\mathrm{x} 400$; scale bar=50 $\mu$ m]. The CONT group shows the standard structure of glomerular capillaries and tubular epithelium; the ATO group exhibits the inflammatory cell infiltration in the renal interstitial, glomeruli dilation and hyperemia; the MgIG treatment group shows a standard structure; and the H-MgIG and L-MgIG groups alleviate renal morphological alterations. (B) A boxplot was used to depict the kidney injury scores, in which the band inside the box represents the median, and the bottom and top of the box represent the lower and upper quartiles, respectively. The circle and star represent the largest and smallest data respectively. Values are demonstrated as median \pm range, $\mathrm{n}=10 .{ }^{* *} \mathrm{P}<0.01$ vs. CONT group; ${ }^{\# \#} \mathrm{P}<0.01,{ }^{\#} \mathrm{P}<0.05$ vs. ATO group. MgIG, magnesium isoglycyrrhizinate; CONT, control group; ATO, arsenic trioxide group; $\mathrm{H}-\mathrm{MgIG}$, high MgIG group (50 mg/kg/day); L-MgIG, low MgIG group (25 mg/kg/day).

$\mathrm{kg} \mathrm{MgIG} \mathrm{showed} \mathrm{no} \mathrm{prominent} \mathrm{difference} \mathrm{in} \mathrm{the} \mathrm{serum} \mathrm{and}$ kidney tissues expression levels of SOD, MDA, CAT and GSH.
Therefore, MgIG treatment may relieve ATO-induced oxidative stress. 

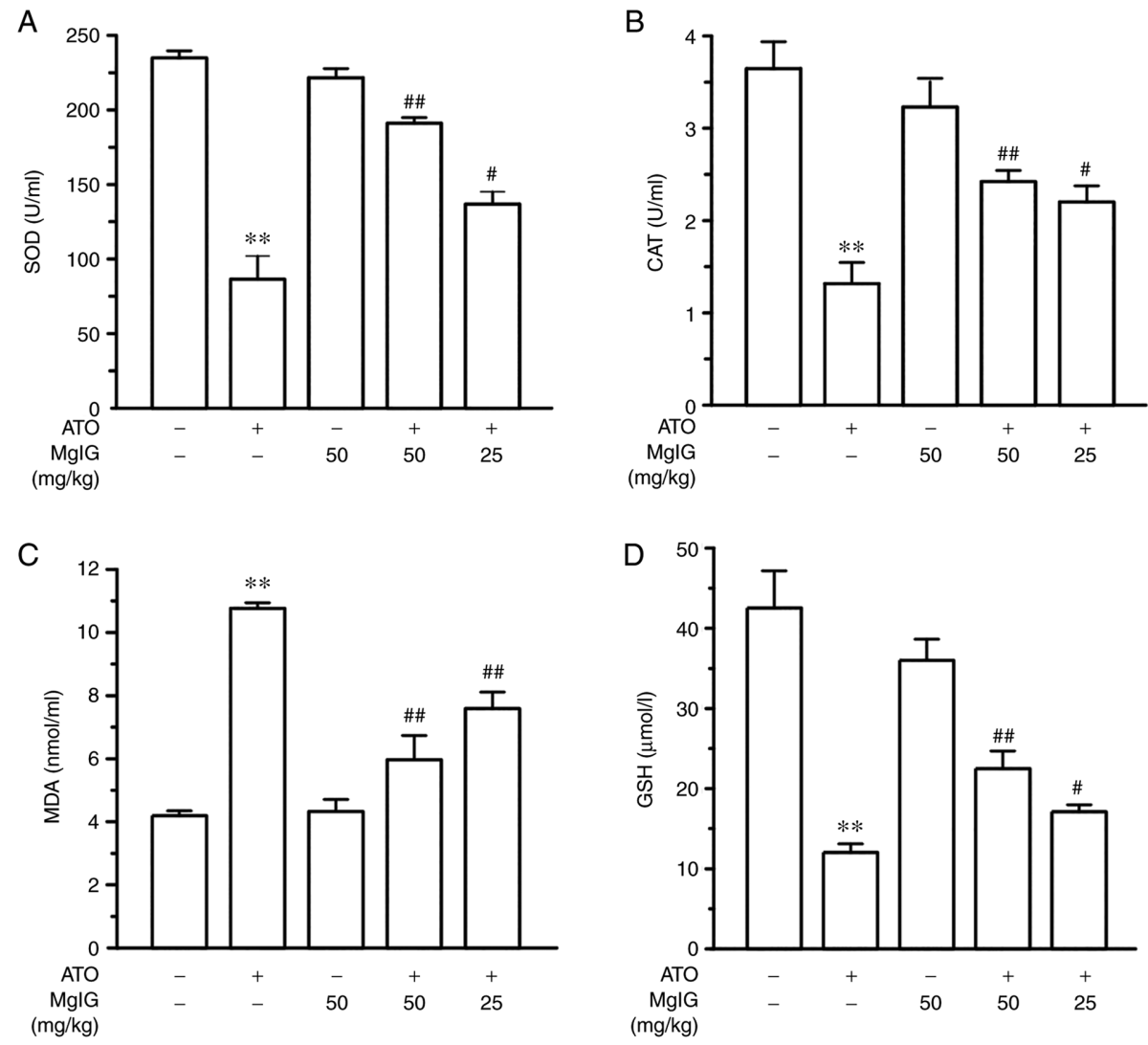

Figure 4. Effects of MgIG on the expression levels of oxidative stress markers (A) SOD, (B) CAT, (C) MDA and (D) GSH in serum. Values are demonstrated as mean \pm SEM. $n=10 .{ }^{* *} \mathrm{P}<0.01$ vs. CONT group; ${ }^{\# \prime} \mathrm{P}<0.01,{ }^{~} \mathrm{P}<0.05$ vs. ATO group. MgIG, magnesium isoglycyrrhizinate; SOD, superoxide dismutase; CAT, catalase; MDA, malondialdehyde; GSH, glutathione; CONT, control group; ATO, arsenic trioxide group.
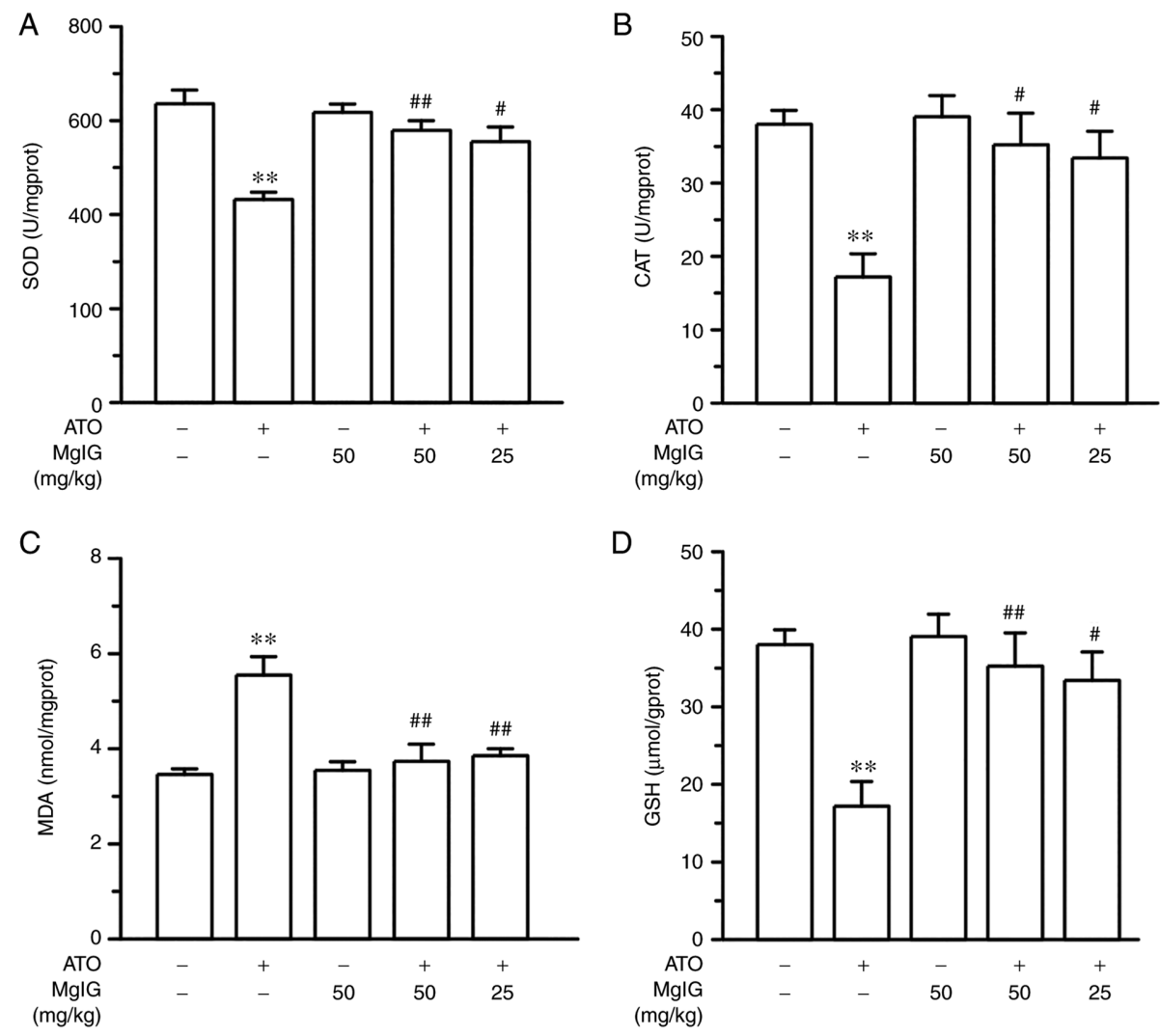

Figure 5. Effects of MgIG on the expression levels of oxidative stress markers (A) SOD, (B) CAT, (C) MDA and (D) GSH in renal tissues. Values are demonstrated as mean \pm SEM. $n=10$. ${ }^{* *} \mathrm{P}<0.01$ vs. CONT group; ${ }^{\# \#} \mathrm{P}<0.01,{ }^{\sharp} \mathrm{P}<0.05$ vs. ATO group. MgIG, magnesium isoglycyrrhizinate; SOD, superoxide dismutase; CAT, catalase; MDA, malondialdehyde; GSH, glutathione; CONT, control group; ATO, arsenic trioxide group. 
A

CONT
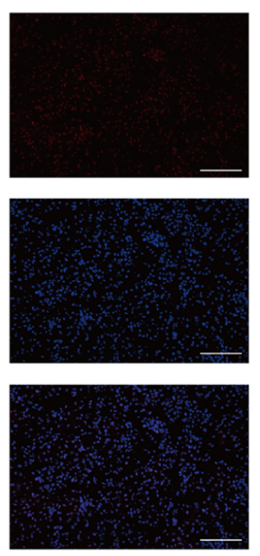

ATO
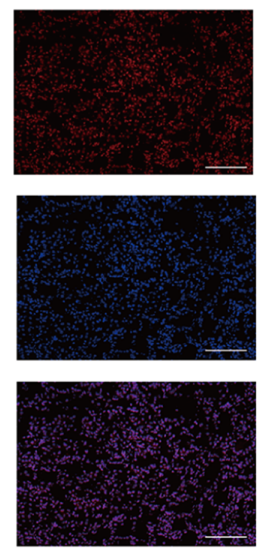

MgIG
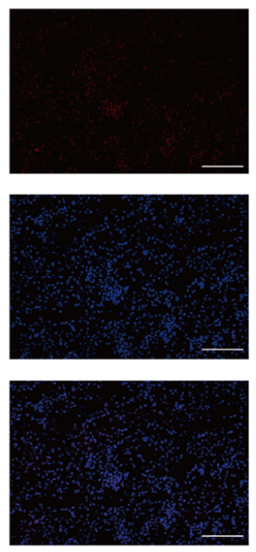
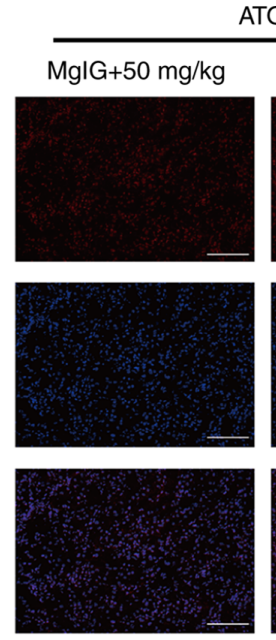

ATO

$\mathrm{MglG}+25 \mathrm{mg} / \mathrm{kg}$

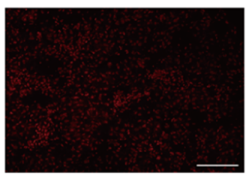

DHE Probe

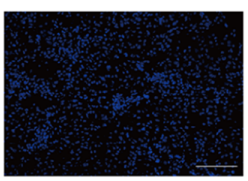

DAPI

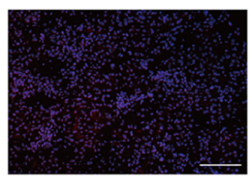

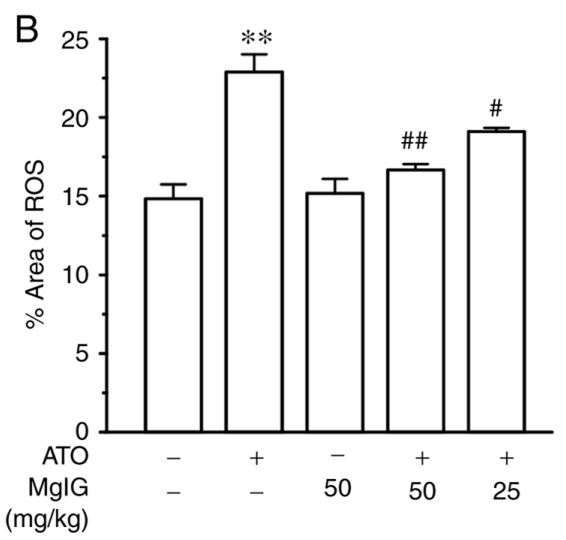

Figure 6. Effects of MgIG on the ROS levels in mice [(A) x200 magnification, scale bar=100 $\mu$ m]. (B) ROS-positive cell area percentages in each group are shown. Values are demonstrated as mean \pm SEM. $n=6{ }^{* *} \mathrm{P}<0.01$ vs. CONT group; ${ }^{\# \#} \mathrm{P}<0.01,{ }^{\#} \mathrm{P}<0.05$ vs. ATO group. MgIG, magnesium isoglycyrrhizinate; ROS, reactive oxygen species; CONT, control group; ATO, arsenic trioxide group.
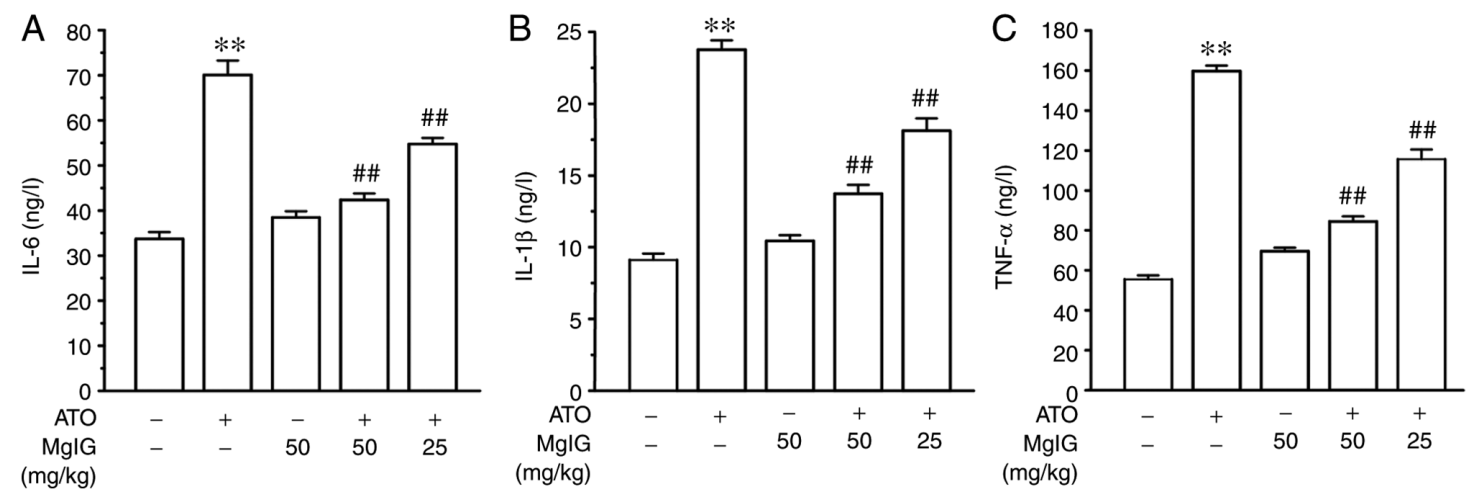

Figure 7. Effects of MgIG on the expression levels in renal tissues of IL-6 (A), IL-1 $\beta$ (B) and TNF- $\alpha$ (C). Values are demonstrated as mean \pm SEM. $n=10$. ${ }^{* *} \mathrm{P}<0.01$ vs. CONT group; ${ }^{\# /} \mathrm{P}<0.01$ vs. ATO group. MgIG, magnesium isoglycyrrhizinate; IL, interleukin; TNF, tumor necrosis factor; CONT, control group; ATO, arsenic trioxide group.

Effects of MgIG on ROS production in ATO-treated mice. As seen in Fig. 6, the kidney slices of mice were stained with a fluorescent dye that identified ROS. The ATO group had substantially greater ROS generation than the CONT group $(\mathrm{P}<0.01)$, whereas no significant changes were found between the CONT group and the MgIG group. In contrast to the ATO group, the H-MgIG and L-MgIG groups had lower ROS generation $(\mathrm{P}<0.05$ or $\mathrm{P}<0.01)$. Thus, MgIG treatment may reduce the ROS production caused by ATO.
Effects of MgIG on IL-6, IL-1 $\beta$ and TNF- $\alpha$ expression. Compared with the CONT group, the ATO group showed increase in IL-6, IL-1 $\beta$ and TNF- $\alpha$ expressions, as shown in Fig. $7(\mathrm{P}<0.01)$. MgIG alone treated mice did not show significant differences when compared to the CONT group. Nevertheless, the levels of IL-6, IL- $1 \beta$ and TNF- $\alpha$ in the H-MgIG and L-MgIG groups were decreased compared with the ATO groups $(\mathrm{P}<0.01)$. MgIG may have inhibitory effects on ATO-induced inflammatory reaction. 
A

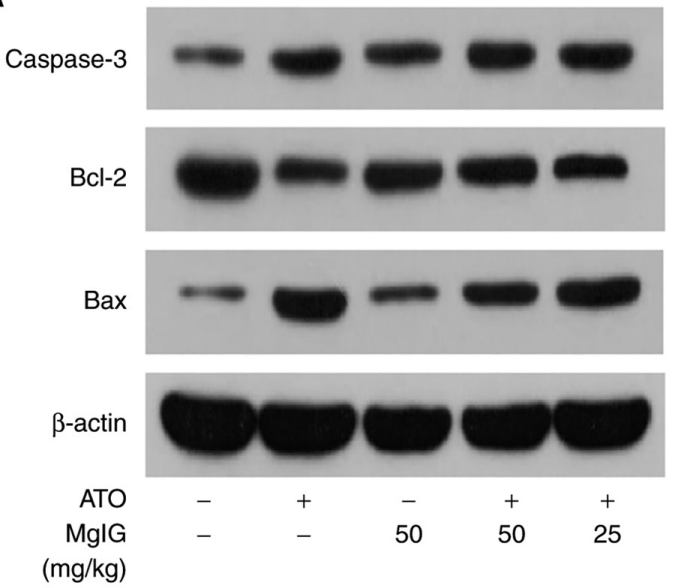

C

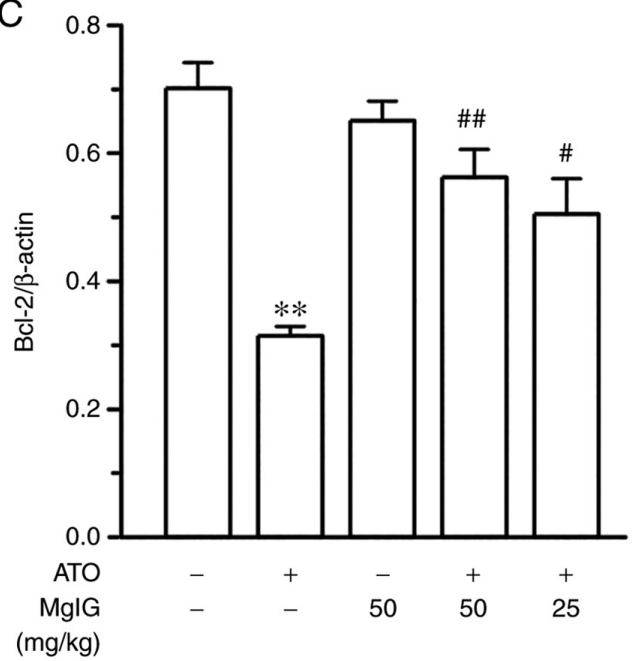

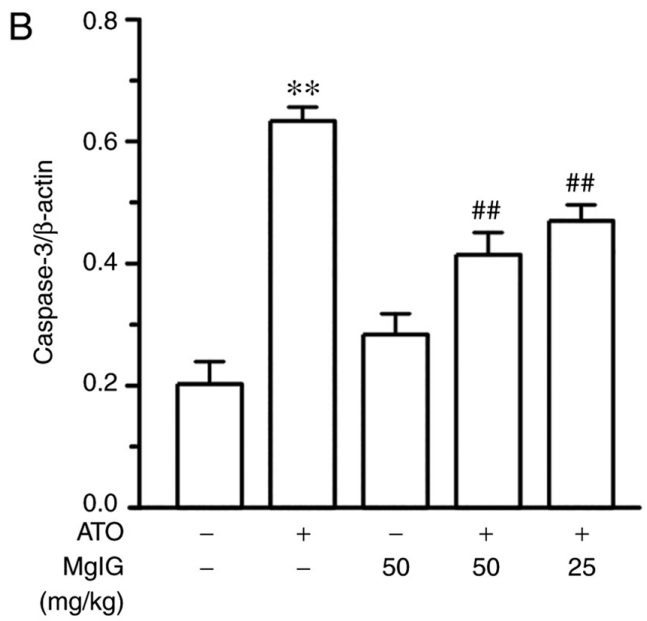

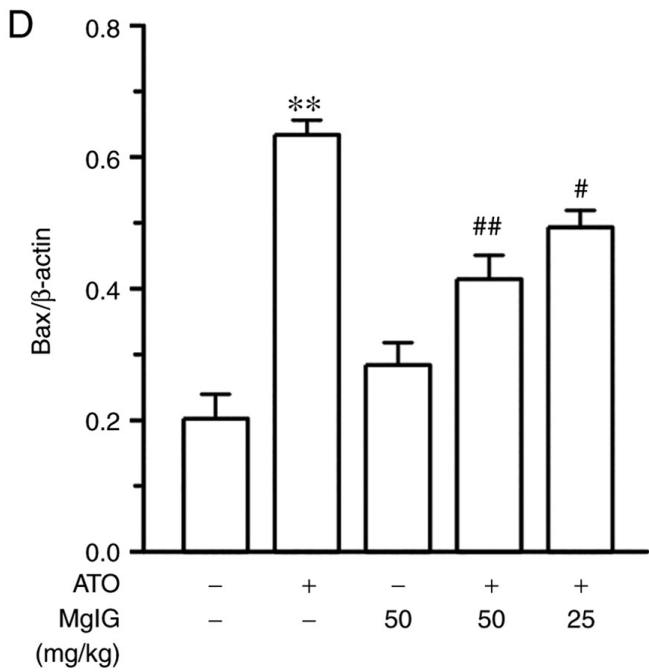

Figure 8. Effects of MgIG on the expression levels of apoptosis indexes were determined by western blot analysis. (A) For each group, the fluorescence intensity of (B) caspase-3, (C) Bcl-2 and (D) Bax was analyzed by normalizing to $\beta$-actin. Values are demonstrated as mean \pm SEM. $n=3$. ${ }^{* *}<<0.01$ vs. CONT group; ${ }^{\# \#} \mathrm{P}<0.01,{ }^{\#} \mathrm{P}<0.05$ vs. ATO group. MgIG, magnesium isoglycyrrhizinate; Bcl-2, B-cell lymphoma 2; Bax, Bcl-2 associated X; CONT, control group; ATO, arsenic trioxide group.

Effects of MgIG on caspase-3, Bcl-2 and Bax expression. In the ATO group, protein expression levels of caspase- 3 and Bax were upregulated, while Bcl-2 protein expression was downregulated, compared with the CONT group as show in Fig. 8 $(\mathrm{P}<0.01)$. However, the expression levels of caspase-3 and Bax were decreased and Bcl-2 expression level was significantly elevated in the L-MgIG group and $\mathrm{H}-\mathrm{MgIG}$ group compared with the ATO group $(\mathrm{P}<0.05$ or $\mathrm{P}<0.01)$. The expression levels of apoptotic proteins in $\mathrm{MgIG}$ alone treated mice did not show significant differences when compared to the CONT group. Thus, MgIG may alleviate the increase in the expression of pro-apoptosis indicators induced by ATO.

Effects of MgIG on TLR $4 / N F-\kappa B$ expression. As shown in Fig. 9, compared with the CONT group, the expressions of TLR4, p-NF- $\kappa$ B, NF- $\kappa \mathrm{B}$ and $\mathrm{p}-\mathrm{NF}-\kappa \mathrm{B} / \mathrm{NF}-\kappa \mathrm{B}$ in the ATO group were markedly higher $(\mathrm{P}<0.01)$. TLR4, p-NF- $\mathrm{B}$, $\mathrm{NF}-\kappa \mathrm{B}$ and $\mathrm{p}-\mathrm{NF}-\kappa \mathrm{B} / \mathrm{NF}-\kappa \mathrm{B}$ expression levels in the $\mathrm{H}-\mathrm{MgIG}$ and L-MgIG groups were lower compared with ATO group $(\mathrm{P}<0.05$ or $\mathrm{P}<0.01)$. The expressions of TLR4, $\mathrm{p}-\mathrm{NF}-\kappa \mathrm{B}$, $\mathrm{NF}-\kappa \mathrm{B}$ and $\mathrm{p}-\mathrm{NF}-\kappa \mathrm{B} / \mathrm{NF}-\kappa \mathrm{B}$ in the MgIG group and CONT group demonstrated no significant difference.

\section{Discussion}

Arsenic, a trivalent inorganic arsenic, also is a worldwide environmental pollutant and a human carcinogen $(6,37,38)$. As MgIG has good lipophilicity, it easily penetrates the cell membrane to bind to the receptor protein and the target cell receptor of steroid hormones, thereby exerting hormonal effects (such as remission lipid metabolism disorder) (24,39).

The present study demonstrated that MgIG can ameliorate ATO-induced kidney damage in mice. In addition to modifying the ATO-mediated increase in BUN and CRE, oxidative stress and inflammatory cytokines were alleviated following MgIG treatment. The hematoxylin and eosin-stained kidney tissues of ATO-treated mice revealed abnormal kidney morphology and the kidney tissues of medication treatment groups showed slight anomaly, which indicted MgIG may possess the protective effect on kidney injury.

The kidney is the main organ of body used for arsenic excretion and also a principal site for arsenic accumulation. The present study found that MgIG could improve the pathological damage of the kidney and mitigate the abnormal variation of serum BUN, serum CRE, urinary CRE and 
A

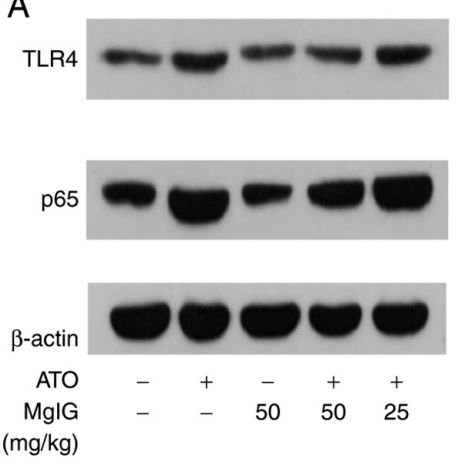

D
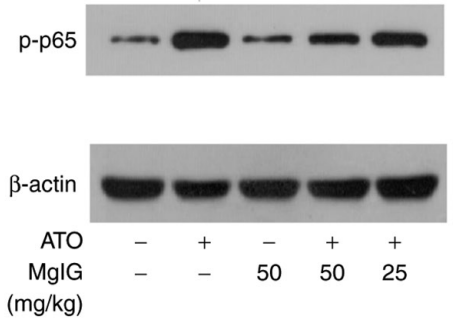

B
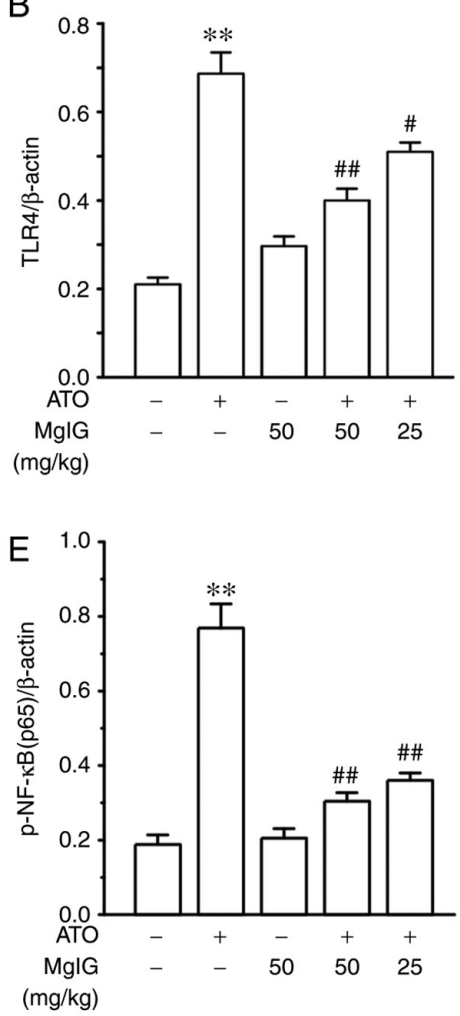

C

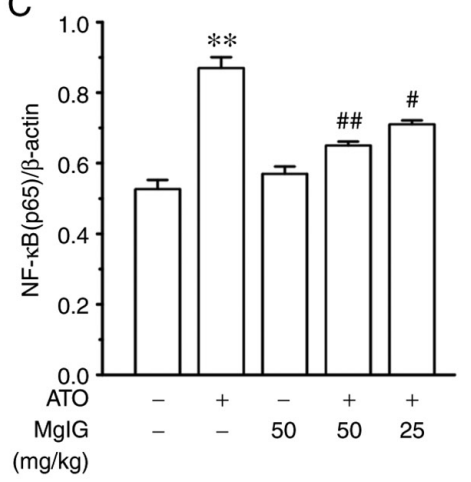

$\mathrm{F}$

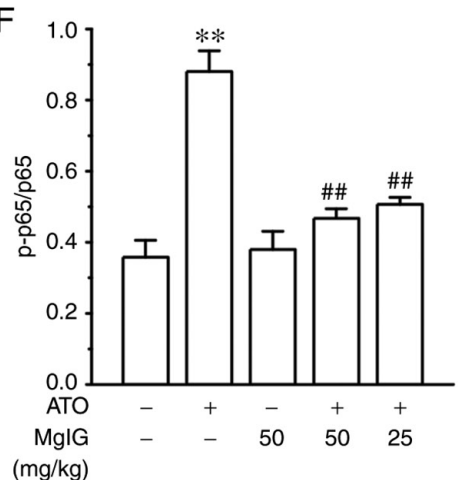

Figure 9. Effects of MgIG on the expression levels of TLR4, NF- $\kappa B$ (p65) and p-NF- $\mathrm{B}$ (p-p65). (A and D) For each group, the intensity of (B) TLR4, (C) NF- $\kappa$ B

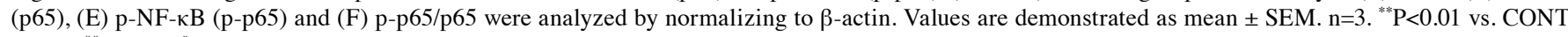
group; ${ }^{\# \#} \mathrm{P}<0.01,{ }^{\#} \mathrm{P}<0.05$ vs. ATO group. MgIG, magnesium isoglycyrrhizinate; TLR4, Toll-like receptor-4; NF- $\kappa \mathrm{B}$, nuclear factor- $\kappa \mathrm{B}$; $\mathrm{p}-$, phosphorylated; CONT, control group; ATO, arsenic trioxide group.

CRE clearance. These markers are typically end-products of nitrogenous compounds and protein metabolism and they are used as biochemical indicators for detecting renal function $(19,40,41)$. The considerable restoration of serum BUN, serum CRE, urinary CRE and CRE clearance activity levels showed that MgIG protected the kidney from the damage from ATO. However, the content of serum BUN, serum CRE, urinary CRE and CRE clearance in MgIG alone administered mice did not change abnormally. In conclusion, treatment of MgIG could alleviate ATO-induced nephrotoxicity.

Studies report that one of the mechanisms of nephrotoxicity induced by ATO is the excessive production of oxidative stress (42). In addition, its anticancer effects are also associated with abnormal oxidative stress (43). One of the ways to mitigate the oxidative stress caused by superfluous ROS is to augment the synergistic effects of antioxidant enzymes (GSH, SOD and CAT) (44). This is consistent with the conclusion of the present study that $\mathrm{MgIG}$ enhanced the activities of antioxidant enzymes and decreased the content of MDA and the production of ROS. In addition, the activities of GSH, SOD and CAT and the production of ROS and MDA in the MgIG group showed no significant change compared with those of the CONT group. As ATO can stimulate ROS production during the process of metabolic activation and ROS can devastate the structure of cells, it causes significant inflammation and ultimately leads to cell apoptosis (45). Therefore, ATO can further exacerbate nephrotoxicity by inducing oxidative stress and inflammation (46). The present study showed that the ATO group had the highest ROS content, which generated kidney damage. ROS can also activate all types of signaling pathways (such as TLR4/NF- $\kappa$ B pathway) (18).

Pro-inflammatory cytokines serve a significant position in the pathogenesis of various inflammatory illnesses (47). $\mathrm{TNF}-\alpha$ is involved in cisplatin-induced nephrotoxicity (48). The activation of NF- $\kappa \mathrm{B}$ can stimulate the transcription of IL-6, IL-1 $\beta$ and TNF- $\alpha$ genes, thereby aggravating the inflammatory response $(47,49)$. The present study found that the levels of IL-6, IL-1 $\beta$ and TNF- $\alpha$ in renal tissues were considerably elevated in the ATO group compared with the CONT group, while treatment with MgIG lessened the expressions of inflammatory factors, illustrating the anti-inflammatory effect of $\operatorname{MgIG}(50,51)$.

Caspases and Bcl-2 family proteins (such as Bcl-2-like proteins, Bax-like proteins) are regulators of apoptotic signaling pathway (52). Bcl-2 is a membrane protein with anti-apoptotic effect and can restrain the activation of caspase-3. Bax is a death-promoting molecule and induces apoptosis (53). In the present study the ATO group demonstrated increased expression levels of caspase- 3 and Bax and a decreased level of Bcl-2. However, the expression of apoptotic protein did not significantly change in the mice treated with MgIG alone. Thus, MgIG treatment slowed down the ATO-induced apoptosis by restoring the caspase-3, Bcl-2 and Bax expression to normal levels.

The activation of TLR can initiate the congenital inflammatory response as TLR4 can bind to a variety of ligands to trigger an inflammatory response $(54,55)$. NF- $\kappa \mathrm{B}$ is a downstream effector of the TLR4 signaling pathway and mediates a variety of inflammatory processes (56). In addition, activation of the NF- $\mathrm{B}$ pathway leads to enhancement of p-p65 


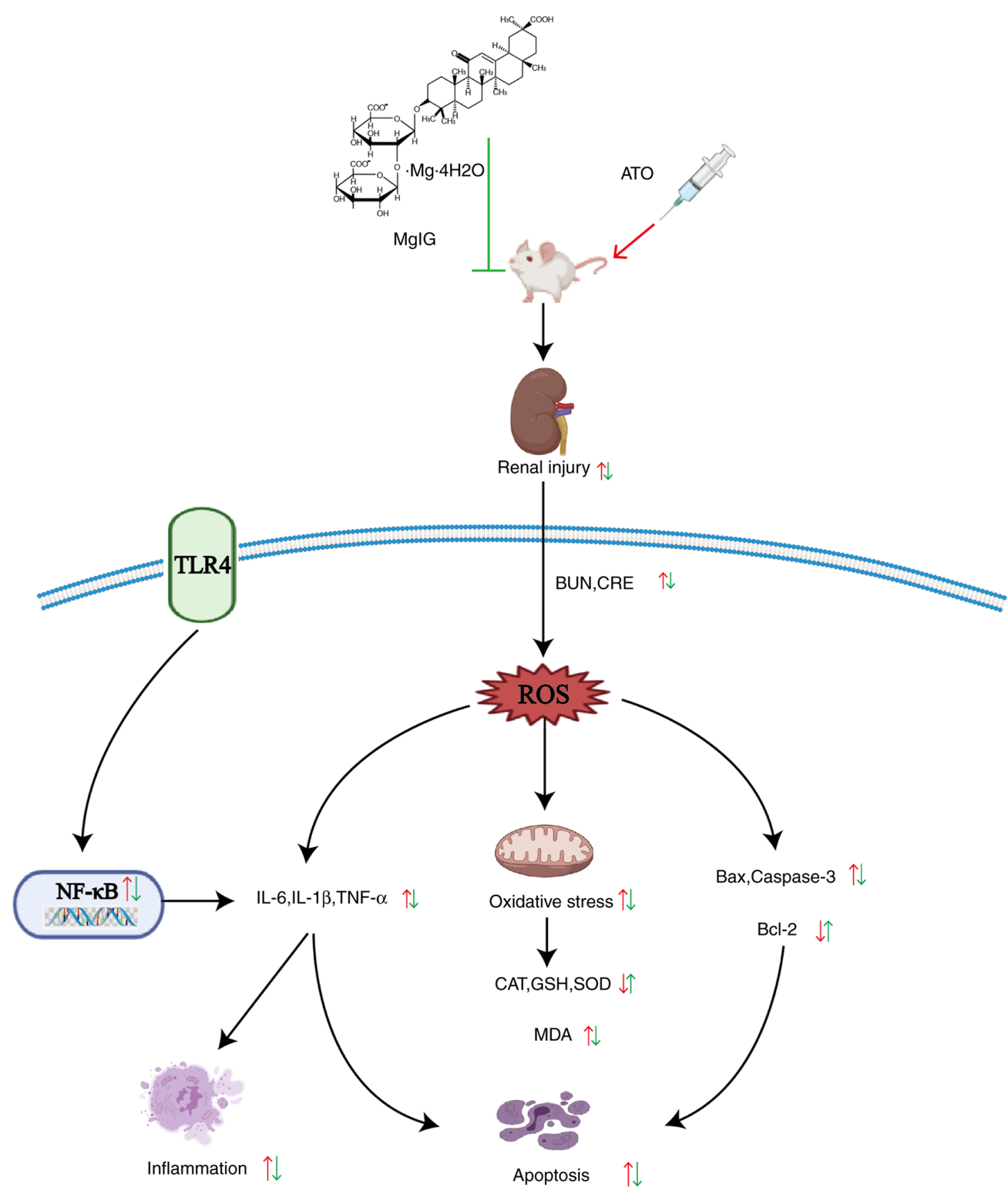

Figure 10. Mechanism of MgIG on ATO-treated nephrotoxicity. MgIG, magnesium isoglycyrrhizinate; ATO, arsenic trioxide; TLR4, Toll-like receptor-4; BUN, blood urea nitrogen; CRE, creatinine; NF- $\mathrm{BB}$, nuclear factor- $\mathrm{kB}$; ROS, reactive oxygen species; IL, interleukin; TNF, tumor necrosis factor; CAT, catalase; GSH, glutathione; SOD, superoxide dismutase; MDA, malondialdehyde; Bax, Bcl-2 associated X; Bcl-2, B-cell lymphoma 2.

and increased expressions of IL-6, IL-1 $\beta$ and TNF- $\alpha$, which eventually leads to the generation of inflammatory reactions in kidney tissue (57). ATO caused a notably increase the expression of $\mathrm{NF}-\kappa \mathrm{B}$ in kidney tissue. However, the mice treated with MgIG did not show a significantly change in the expression of TLR4, p-p65 and p65. Nevertheless, treatment with MgIG downregulated the TLR4 and NF- $\kappa \mathrm{B}$ expression and inhibited the production of pro-inflammatory cytokines. Thus, the protective effect of $\mathrm{MgIG}$ in mice with acute renal injury may be due to its inhibition of TLR4 and NF- $\mathrm{B}$ signaling.

In summary, the present study found that $\mathrm{MgIG}$ may protect against kidney toxicity through its antioxidant, anti-inflammation and anti-apoptosis properties. The results demonstrated that MgIG significantly mitigated ATO-induced nephrotoxicity by decreasing oxidative stress and inflammation, possibly via restraining the TLR4/NF- $\kappa \mathrm{B}$ signaling pathway (Fig. 10). The results indicated that $\mathrm{MgIG}$ could be an effective therapy against ATO-induced nephrotoxicity.
However, in clinical practice, the combination of MgIG with ATO requires further research.

\section{Acknowledgements}

Not applicable.

\section{Funding}

The present study was supported by the Research Foundation of Administration of Traditional Chinese Medicine of Hebei Province, China (grant nos. 2019135 and 2020188).

\section{Availability of data and materials}

The datasets used and/or analyzed during the current study are available from the corresponding author on reasonable request. 


\section{Authors' contributions}

ZWe, ZWu, XC and SG were involved in the conception and planning of the current study. ZWe, XS, QH and YZ performed the experiments. ZWe, QH and XS interpreted the data. ZWe, $\mathrm{YZ}, \mathrm{YW}$ and $\mathrm{XH}$ were involved in the data analysis. $\mathrm{YZ}, \mathrm{YW}$, $\mathrm{XH}$ and $\mathrm{ZWu}$ provided guidance for software and figures. ZWe wrote the original draft. XS, QH, YZ, YW, XH, ZWu, $\mathrm{XC}$ and $\mathrm{SG}$ reviewed and edited the manuscript. $\mathrm{XH}, \mathrm{ZWu}$ and $\mathrm{XC}$ supervised the project. SG identified resources. ZWe and SG confirm the authenticity of all the raw data. All authors have read and approved the final version of the manuscript.

\section{Ethics approval and consent to participate}

All animal procedures were authorized by Hebei Medical University of Chinese Medicine's Animal Care and Ethical Committee (approval no. DWLL2020005).

\section{Patient consent for publication}

Not applicable.

\section{Competing interests}

The authors declare that they have no competing interests.

\section{References}

1. Watanabe T and Hirano S: Metabolism of arsenic and its toxicological relevance. Arch Toxicol 87: 969-979, 2013.

2. Antman KH: Introduction: The history of arsenic trioxide in cancer therapy. Oncologist 6 (Suppl 2): S1-S2, 2001.

3. Bao L and Shi H: Potential molecular mechanisms for combined toxicity of arsenic and alcohol. J Inorg Biochem 104: 1229-1233, 2010.

4. You BR and Park WH: Arsenic trioxide induces human pulmonary fibroblast cell death via increasing ROS levels and GSH depletion. Oncol Rep 28: 749-757, 2012.

5. Simon JP, Parthasarathy M, Nithyanandham S, Katturaja RK, Namachivayam A and Prince SE: Protective effect of the ethanolic and methanolic leaf extracts of madhuca longifolia against diclofenac-induced toxicity in female wistar albino rats. Pharmacol Rep 71: 983-993, 2019.

6. Katturajan R and Evan Prince S: A role of connexin 43 on the drug-induced liver, kidney, and gastrointestinal tract toxicity with associated signaling pathways. Life Sci 280: 119629, 2021.

7. Zhang J, Pan X, Li N, Li X, Wang Y, Liu X, Yin X and Yu Z: Grape seed extract attenuates arsenic-induced nephrotoxicity in rats. Exp Ther Med 7: 260-266, 2014.

8. Yu M, Xue J, Li Y, Zhang W, Ma D, Liu L and Zhang Z: Resveratrol protects against arsenic trioxide-induced nephrotoxicity by facilitating arsenic metabolism and decreasing oxidative stress. Arch Toxicol 87: 1025-1035, 2013.

9. Zhang W, Liu Y, Ge M, Jing J, Chen Y, Jiang H, Yu H, Li N and Zhang Z: Protective effect of resveratrol on arsenic trioxide-induced nephrotoxicity in rats. Nutr Res Pract 8: 220-226, 2014

10. Shi H, Shi X and Liu K: Oxidative mechanism of arsenic toxicity and carcinogenesis. Mol Cell Biochem 255: 67-78, 2004.

11. Lushchak VI: Free radicals, reactive oxygen species, oxidative stress and its classification. Chem Biol Interact 224: 164-175, 2014.

12. Avlas O, Fallach R, Shainberg A, Porat E and Hochhauser E: Toll-like receptor 4 stimulation initiates an inflammatory response that decreases cardiomyocyte contractility. Antioxid Redox Signal 15: 1895-1909, 2011.

13. Tang J, Xu L, Zeng Y and Gong F: Effect of gut microbiota on LPS-induced acute lung injury by regulating the TLR4/NF-kB signaling pathway. Int Immunopharmacol 91: 107272, 2021.
14. Mir SM, Ravuri HG, Pradhan RK, Narra S, Kumar JM, Kuncha M, Kanjilal S and Sistla R: Ferulic acid protects lipopolysaccharide-induced acute kidney injury by suppressing inflammatory events and upregulating antioxidant defenses in Balb/c mice. Biomed Pharmacother 100: 304-315, 2018.

15. Feng D, Wang Y, Liu Y, Wu L, Li X, Chen Y, Chen Y, Chen Y, $\mathrm{Xu} \mathrm{C}$, Yang $\mathrm{K}$ and Zhou T: DC-SIGN reacts with TLR-4 and regulates inflammatory cytokine expression via NF- $\kappa$ B activation in renal tubular epithelial cells during acute renal injury. Clin Exp Immunol 191: 107-115, 2018.

16. Zhong G, Wan F, Ning Z, Wu S, Jiang X, Tang Z, Huang R and $\mathrm{Hu} \mathrm{L}$ : The protective role of autophagy against arsenic trioxide-induced cytotoxicity and ROS-dependent pyroptosis in NCTC-1469 cells. J Inorg Biochem 217: 111396, 2021.

17. Farooq MA, Niazi AK, Akhtar J, Saifullah, Farooq M, Souri Z, Karimi N and Rengel Z: Acquiring control: The evolution of ROS-induced oxidative stress and redox signaling pathways in plant stress responses. Plant Physiol Biochem 141: 353-369, 2019.

18. Roy A, Manna P and Sil PC: Prophylactic role of taurine on arsenic mediated oxidative renal dysfunction via MAPKs/ NF-kappaB and mitochondria dependent pathways. Free Radic Res 43: 995-1007, 2009.

19. Nandi D, Patra RC and Swarup D: Oxidative stress indices and plasma biochemical parameters during oral exposure to arsenic in rats. Food Chem Toxicol 44: 1579-1584, 2006.

20. Zhang K, Zhao P, Guo G, Guo Y, Tian L, Sun X, Li S, He Y, Sun Y, Chai H, et al: Arsenic trioxide attenuates NF-kB and cytokine mRNA levels in the livers of cocks. Biol Trace Elem Res 170: 432-437, 2016.

21. Zheng B, Yang Y, Li J, Li J, Zuo S, Chu X, Xu S, Ma D and Chu L: Magnesium isoglycyrrhizinate alleviates arsenic trioxide-induced cardiotoxicity: Contribution of Nrf2 and TLR4/ NF-кB signaling pathway. Drug Des Devel Ther 15: 543-556, 2021.

22. Yang YZ, Liu ZH, Wang SC, Zhang XQ, Xu HJ, Yang L and Kong LD: Magnesium isoglycyrrhizinate alleviates fructose-induced liver oxidative stress and inflammatory injury through suppressing NOXs. Eur J Pharmacol 883: 173314, 2020.

23. Zhao Z, Tang Z, Zhang W, Liu J and Li B: Magnesium isoglycyrrhizinate protects against renal-ischemia-reperfusion injury in a rat model via anti-inflammation, anti-oxidation and anti-apoptosis. Mol Med Rep 16: 3627-3633, 2017.

24. Zheng J, Wu G, Hu GX, Peng YZ and Xiong XJ: Protective effects against and potential mechanisms underlying the effect of magnesium isoglycyrrhizinate in hypoxia-reoxygenation injury in rat liver cells. Genet Mol Res 14: 15453-15461, 2015.

25. Ma D, Zhang J, Zhang Y, Zhang X, Han X, Song T, Zhang Y and Chu L: Inhibition of myocardial hypertrophy by magnesium isoglycyrrhizinate through the TLR4/NF- $\kappa \mathrm{B}$ signaling pathway in mice. Int Immunopharmacol 55: 237-244, 2018.

26. Liao Y, Tan RZ, Li JC, Liu TT, Zhong X, Yan Y, Yang JK, Lin X, Fan JM and Wang L: Isoliquiritigenin attenuates UUO-induced renal inflammation and fibrosis by inhibiting mincle/Syk/ NF-Kappa B signaling pathway. Drug Des Devel Ther 14: $1455-1468,2020$

27. Percie du Sert N, Ahluwalia A, Alam S, Avey MT, Baker M, Browne WJ, Clark A, Cuthill IC, Dirnagl U, Emerson M, et al. Reporting animal research: Explanation and elaboration for the ARRIVE guidelines 2.0. PLoS Biol 18: e3000411, 2020.

28. Liu M, Zheng B, Liu P, Zhang J, Chu X, Dong C, Shi J, Liang Y, Chu L, Liu Y and Han X: Exploration of the hepatoprotective effect and mechanism of magnesium isoglycyrrhizinate in mice with arsenic trioxide-induced acute liver injury. Mol Med Rep 23: 438, 2021.

29. Li SG, Xu SZ, Niu Q, Ding YS, Pang LJ, Ma RL, Jing MX, Wang K, Ma XM,Feng GL, et al: Lutein alleviates arsenic-induced reproductive toxicity in male mice via Nrf2 signaling. Hum Exp Toxicol 35: 491-500, 2016.

30. Jiang W, Chen Q, Li P, Lu Q, Pei X, Sun Y, Wang G and Hao K: Magnesium Isoglycyrrhizinate attenuates lipopolysaccharide-induced depressive-like behavior in mice. Biomed Pharmacother 86: 177-184, 2017.

31. Jiang W, Guo H, Su D, Xu H, Gu H and Hao K: Ameliorative effect of magnesium isoglycyrrhizinate on hepatic encephalopathy by epirubicin. Int Immunopharmacol 75: 105774, 2019.

32. Laferriere CA, Leung VS and Pang DS: Evaluating intrahepatic and intraperitoneal sodium pentobarbital or ethanol for mouse euthanasia. J Am Assoc Lab Anim Sci 59: 264-268, 2020. 
33. Kim HY, Yokozawa T, Nakagawa T and Sasaki S: Protective effect of gamma-aminobutyric acid against glycerol-induced acute renal failure in rats. Food Chem Toxicol 42: 2009-2014, 2004.

34. Tonomura Y, Yamamoto E, Kondo C, Itoh A, Tsuchiya N, Uehara T and Baba T: Amphotericin B-induced nephrotoxicity: Characterization of blood and urinary biochemistry and renal morphology in mice. Hum Exp Toxicol 28: 293-300, 2009.

35. Elliott AC and Hynan LS: A SAS(®) macro implementation of a multiple comparison post hoc test for a Kruskal-Wallis analysis. Comput Methods Programs Biomed 102: 75-80, 2011.

36. Yazdani M: Concerns in the application of fluorescent probes DCDHF-DA, DHR 123 and DHE to measure reactive oxygen species in vitro. Toxicol In Vitro 30: 578-582, 2015.

37. Ferreccio C, Smith AH, Durán V, Barlaro T, Benítez H, Valdés R, Aguirre JJ, Moore LE, Acevedo J, Vásquez MI, et al: Case-control study of arsenic in drinking water and kidney cancer in uniquely exposed Northern Chile. Am J Epidemiol 178: 813-818, 2013.

38. Surdu S, Fitzgerald EF, Bloom MS, Boscoe FP, Carpenter DO, Haase RF, Gurzau E, Rudnai P, Koppova K, Févotte J, et al: Occupational exposure to arsenic and risk of nonmelanoma skin cancer in a multinational European study. Int J Cancer 133: 2182-2191, 2013

39. Zhao XJ, Yang YZ, Zheng YJ, Wang SC, Gu HM, Pan Y, Wang SJ, $\mathrm{Xu} \mathrm{HJ}$ and Kong LD: Magnesium isoglycyrrhizinate blocks fructose-induced hepatic NF-אB/NLRP3 inflammasome activation and lipid metabolism disorder. Eur J Pharmacol 809: 141-150, 2017.

40. Gora RH, Kerketta P, Baxla SL, Toppo R, Prasad R, Patra PH and Roy BK: Ameliorative effect of tephrosia purpurea in arsenic-induced nephrotoxicity in rats. Toxicol Int 21: 78-83, 2014.

41. Wang X, Zhao H, Shao Y, Wang P, Wei Y, Zhang W, Jiang J, Chen Y and Zhang Z: Nephroprotective effect of astaxanthin against trivalent inorganic arsenic-induced renal injury in wistar rats. Nutr Res Pract 8: 46-53, 2014.

42. Dutta S, Saha S, Mahalanobish S, Sadhukhan P and Sil PC: Melatonin attenuates arsenic induced nephropathy via the regulation of oxidative stress and inflammatory signaling cascades in mice. Food Chem Toxicol 118: 303-316, 2018.

43. Kumar S, Yedjou CG and Tchounwou PB: Arsenic trioxide induces oxidative stress, DNA damage, and mitochondrial pathway of apoptosis in human leukemia (HL-60) cells. J Exp Clin Cancer Res 33: 42, 2014.

44. Schieber M and Chandel NS: ROS function in redox signaling and oxidative stress. Curr Biol 24: R453-R462, 2014.

45. Wang Y, Zhao H, Shao Y, Liu J, Li J and Xing M: Copper or/ and arsenic induce oxidative stress-cascaded, nuclear factor kappa B-dependent inflammation and immune imbalance, trigging heat shock response in the kidney of chicken. Oncotarget 8: 98103-98116, 2017.

46. Lushchak VI: Classification of oxidative stress based on its intensity. EXCLI J 13: 922-937, 2014.
47. Kumar P, Sulakhiya K, Barua CC and Mundhe N: TNF- $\alpha$, IL-6 and IL-10 expressions, responsible for disparity in action of curcumin against cisplatin-induced nephrotoxicity in rats. Mol Cell Biochem 431: 113-122, 2017.

48. Jiang L, Liu Y, He P, Chen J, Liu S and Tan N: Geraniin ameliorates cisplatin-induced nephrotoxicity in mice. Free Radic Res 50: 813-819, 2016.

49. Abou-Hany HO, Atef H, Said E, Elkashef HA and Salem HA: Crocin mediated amelioration of oxidative burden and inflammatory cascade suppresses diabetic nephropathy progression in diabetic rats. Chem Biol Interact 284: 90-100, 2018.

50. Xie C, Li X, Wu J, Liang Z, Deng F, Xie W, Zhu M, Zhu J, Zhu W, Geng S and Zhong C: Anti-inflammatory activity of magnesium isoglycyrrhizinate through inhibition of phospholipase A2/Arachidonic acid pathway. Inflammation 38: 1639-1648, 2015

51. Lin G, Nnane IP and Cheng TY: The effects of pretreatment with glycyrrhizin and glycyrrhetinic acid on the retrorsine-induced hepatotoxicity in rats. Toxicon 37: 1259-1270, 1999.

52. Wang YL, Wang CY, Zhang BJ and Zhang ZZ: Shenfu injection suppresses apoptosis by regulation of Bcl-2 and caspase-3 during hypoxia/reoxygenation in neonatal rat cardiomyocytes in vitro. Mol Biol Rep 36: 365-370, 2009.

53. Pratheeshkumar P and Kuttan G: Oleanolic acid induces apoptosis by modulating $\mathrm{p} 53, \mathrm{Bax}, \mathrm{Bcl}-2$ and caspase- 3 gene expression and regulates the activation of transcription factors and cytokine profile in B16F. J Environ Pathol Toxicol Oncol 30: 21-31, 2011.

54. Lagos D, Vart RJ, Gratrix F, Westrop SJ, Emuss V, Wong PP, Robey R, Imami N, Bower M, Gotch F and Boshoff C: Toll-like receptor 4 mediates innate immunity to Kaposi sarcoma herpesvirus. Cell Host Microbe 4: 470-483, 2008.

55. Du Y, Qian B, Gao L, Tan P, Chen H, Wang A, Zheng T, Pu S, $\mathrm{Xia} X$ and $\mathrm{Fu} \mathrm{W}$ : Aloin preconditioning attenuates hepatic ischemia/reperfusion injury via inhibiting TLR4/MyD88/NF- $\mathrm{B}$ signal pathway in vivo and in vitro. Oxid Med Cell Longev 2019: $3765898,2019$.

56. Elsharkawy AM and Mann DA: Nuclear factor-kappaB and the hepatic inflammation-fibrosis-cancer axis. Hepatology 46: 590-597, 2007.

57. Song J, Fan H, Li H, Ding H, Lv Q and Hou S: Zingerone ameliorates lipopolysaccharide-induced acute kidney injury by inhibiting Toll-like receptor 4 signaling pathway. Eur J Pharmacol 772: 108-114, 2016.

This work is licensed under a Creative Commons Attribution-NonCommercial-NoDerivatives 4.0 International (CC BY-NC-ND 4.0) License. 